Invited Paper

\title{
Optical frequency standards based on mercury and aluminum ions
}

\author{
W. M. Itano, J. C. Bergquist, A. Brusch, S. A. Diddams, T. M. Fortier, T. P. Heavner, L. \\ Hollberg, D. B. Hume, S. R. Jefferts, L. Lorini, T. E. Parker, T. Rosenband, and J. E. \\ Stalnaker \\ Time and Frequency Division, National Institute of Standards and Technology, Boulder, CO \\ 80305, USA
}

\begin{abstract}
Single-trapped-ion frequency standards based on a $282 \mathrm{~nm}$ transition in ${ }^{199} \mathrm{Hg}^{+}$and on a $267 \mathrm{~nm}$ transition in ${ }^{27} \mathrm{Al}^{+}$have been developed at NIST over the past several years. Their frequencies are measured relative to each other and to the NIST primary frequency standard, the NIST-F1 cesium fountain, by means of a self-referenced femtosecond laser frequency comb. Both ion standards have demonstrated instabilities and inaccuracies of less than $1 \times 10^{-16}$.
\end{abstract}

Keywords: aluminum, atomic clocks, frequency standards, ion traps, mercury

\section{INTRODUCTION}

Optical frequency standards based on the mercury ion and, more recently, the aluminum ion are under development at the National Institute of Standards and Technology. Both are based on narrow optical transitions in single trapped ions, and both have demonstrated very good frequency stabilities and reproducibilities. By means of a self-referenced femtosecond laser frequency comb, their frequencies can be compared to a microwave frequency standard and to each other. A microwave-to-optical frequency comparison yields a measurement of the optical frequency in terms of the SI (International System of Units) second, which is based on the cesium hyperfine frequency. According to our estimates of systematic uncertainties, both the mercury and aluminum standards are more reproducible than current primary cesium frequency standards. This places a limitation on the accuracy of measurements of the mercury and aluminum optical resonance frequencies in terms of the SI second. However, measurements of the ratio of the mercury and aluminum frequencies are not subject to this limitation. Measurements of this ratio as a function of time place limits on the variation of the fundamental constants, such as the fine-structure constant $\alpha$.

Since some details of the mercury and aluminum ion frequency standards have already been published, this article will emphasize subjects that that have not been discussed in detail previously.

\section{MERCURY ION FREQUENCY STANDARD}

The first proposal to use the $282 \mathrm{~nm}$ transition from the ground $5 d^{10} 6 s^{2} S_{1 / 2}$ state to the metastable $5 d^{9} 6 s^{2}{ }^{2} D_{5 / 2}$ state of $\mathrm{Hg}^{+}$as an optical frequency was made by Bender et al. ${ }^{1}$ The metastable state has a natural lifetime of around $90 \mathrm{~ms},{ }^{2-5}$ giving this transition a $Q$ of around $6 \times 10^{14}$. Work began at NIST on mercury ion optical frequency standards several years later. The transition was first observed by Doppler-free two-photon absorption of a cloud of trapped ${ }^{198} \mathrm{Hg}^{+}$ions. ${ }^{2}$ The transition was later observed in a single trapped ${ }^{198} \mathrm{Hg}^{+}$ion by singlephoton electric-quadrupole absorption. ${ }^{6}$ Doppler broadening was eliminated for single-photon absorption by confinement of the ion to less than the wavelength of the radiation. ${ }^{7}$ The observed linewidth of about $30 \mathrm{kHz}$

Further author information: (Send correspondence to W.M.I.)

W.M.I.: E-mail: itano@boulder.nist.gov

T.M.F: Also with Los Alamos National Laboratory, P-23 Physics Division MS H803, Los Alamos, NM 87545, USA

L.L.: Permanent address: IEN, Str. delle Cacce 91, 10135 Torino, Italy

J.E.S.: New address: Department of Physics and Astronomy, Oberlin College, Oberlin, OH 44073

Time and Frequency Metrology, edited by R. Jason Jones,

Proc. of SPIE Vol. 6673, 667303, (2007) · 0277-786X/07/\$18 - doi: 10.1117/12.734662

Proc. of SPIE Vol. 6673 667303-1 


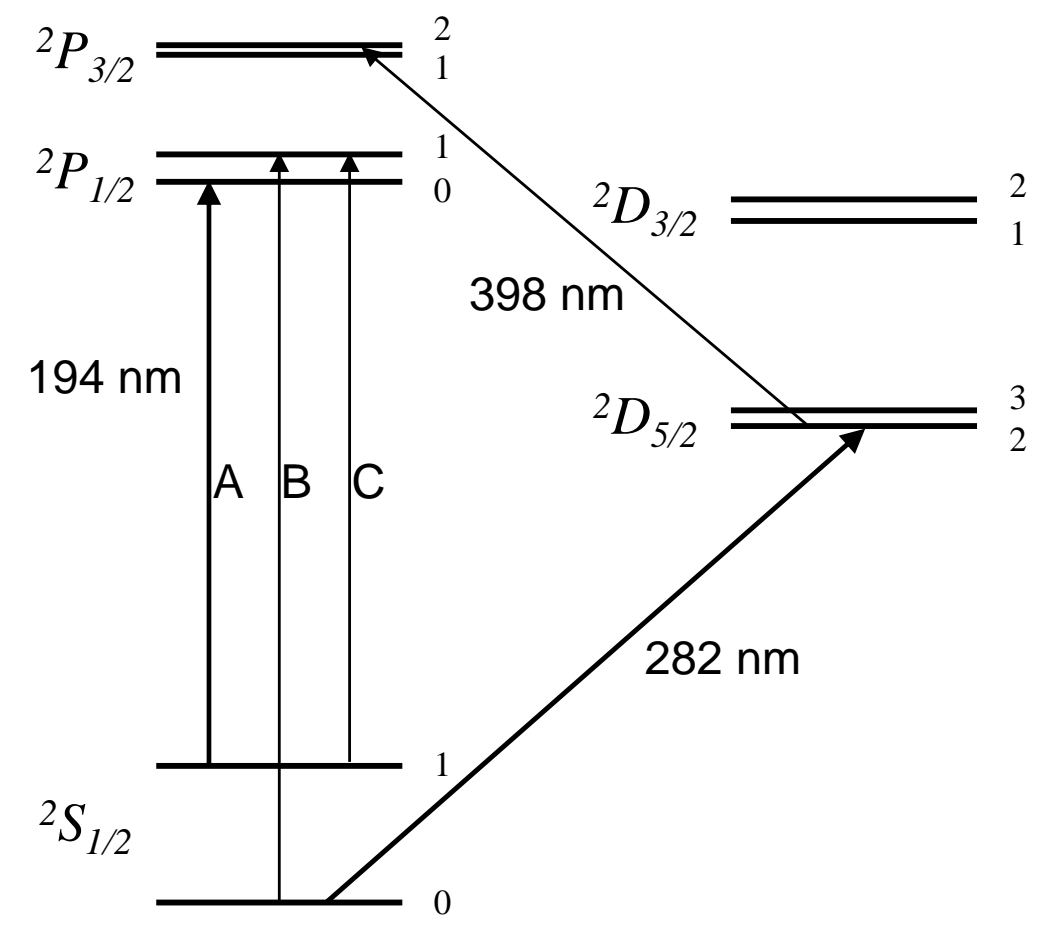

Figure 1. Energy levels of ${ }^{199} \mathrm{Hg}^{+}$. Numbers to the right of the hyperfine energy levels are the values of $F$, the total angular momentum quantum number. Transitions induced by the lasers are indicated by arrows. For clarity, the energy differences between hyperfine levels are expanded relative to the electronic energy differences.

was limited by the laser linewidth and by the magnetic field instability. Further work resulted in narrowing the frequency width of the laser to less than $1 \mathrm{~Hz}^{8}$ Line broadening due the magnetic field was reduced by use of the $(F=0)$ to $\left(F=2, m_{F}=0\right)$ hyperfine-Zeeman component in ${ }^{199} \mathrm{Hg}^{+}$, which has no linear Zeeman shift and a quadratic Zeeman shift of $1.90 \times 10^{4} \mathrm{~Hz} / \mathrm{mT}^{2}$. With these improvements, the $282 \mathrm{~nm}$ resonance was observed with a linewidth as low as $6.7 \mathrm{~Hz} .{ }^{9}$ The frequency of the laser was servo-locked to the atomic resonance so that the apparatus functioned as a frequency standard. With the development of the self-referenced femtosecond laser frequency comb, ${ }^{10-12}$ it became possible to compare the frequency of the $\mathrm{Hg}^{+}$-stabilized laser to microwave or other optical frequency standards. ${ }^{13-18}$

\subsection{State Preparation and Measurement}

The basic methods used for laser cooling, state preparation, and detection of the clock transition have been described previously, ${ }^{9}$ but some additional laser beams are now used to reduce the dead time in the measurement cycle and thereby improve the frequency stability. The energy levels of ${ }^{199} \mathrm{Hg}^{+}$that are relevant to the operation of the frequency standard are shown in Fig. 1. The $194 \mathrm{~nm} 5 d^{10} 6 s^{2} S_{1 / 2}$ to $5 d^{10} 6 p^{2} P_{1 / 2}$ transition is used for Doppler laser cooling and fluorescence detection. The main laser cooling beam is tuned to the $(F=1)$ to $(F=0)$ component, labeled A in Fig. 1. To a first approximation, this is a cycling transition, since decay from $(F=0)$ to $(F=0)$ is forbidden. However, if the magnetic field is low, the ion can be trapped in a nonabsorbing dark state. This can be prevented by applying a large magnetic field, which is undesirable for a frequency standard, or by polarization modulation of the laser. ${ }^{19}$ In earlier work, two beams having different propagation directions but with the same frequency were used. The polarization of one beam was modulated between right and left circular polarization. ${ }^{20}$ More recently, trapping in a dark state has been prevented by irradiating the ion with three noncollinear beams with frequencies differing by several megahertz. ${ }^{16}$ This has enabled reducing the magnetic field to around $8 \mu \mathrm{T}$. To prevent trapping of the ion in the ground $(F=0)$ state, due to off-resonant optical pumping through the ${ }^{2} P_{1 / 2}(F=1)$ state, a weak laser beam tuned to the $(F=0)$ to $(F=1)$ component, labeled B in Fig. 1, is introduced. 
Prior to driving the $282 \mathrm{~nm}$ clock transition, the ion must be prepared in the ${ }^{2} S_{1 / 2}(F=0)$ hyperfine state. Previously, this was done by shutting off the $(F=0)$ to $(F=1) 194 \mathrm{~nm}$ radiation (component B) while leaving on the $(F=1)$ to $(F=0) 194 \mathrm{~nm}$ radiation (component $\mathrm{A})$. This introduced some dead time into the measurement cycle, since around $14 \mathrm{~ms}$ had to be allowed for the ion to be pumped into the $(F=0)$ ground state by off-resonant excitation of the ${ }^{2} P_{1 / 2}(F=1)$. In the current setup, a $194 \mathrm{~nm}$ laser tuned to the $(F=1)$ to $(F=1)$ frequency (component $\mathrm{C}$ ) is introduced to quickly drive the ion from the ${ }^{2} S_{1 / 2}(F=1)$ state to the ${ }^{2} S_{1 / 2}(F=0)$ state, thereby reducing the dead time.

In order to determine whether the ${ }^{2} S_{1 / 2}$ to ${ }^{2} D_{5 / 2} 282 \mathrm{~nm}$ clock transition has been driven, $194 \mathrm{~nm}$ radiation at the $\mathrm{A}$ and $\mathrm{B}$ frequencies is applied. If $194 \mathrm{~nm}$ fluorescence is observed, then the transition did not occur. If the transition did occur, then no fluorescence is observed, and it is necessary to wait $90 \mathrm{~ms}$ on the average for the ion to decay back to the ${ }^{2} S_{1 / 2}$ state before attempting to drive the $282 \mathrm{~nm}$ transition again. In the current setup, a laser tuned to the $398 \mathrm{~nm}^{2} D_{5 / 2}(F=2)$ to ${ }^{2} P_{3 / 2}(F=2)$ transition is used to empty the ${ }^{2} D_{5 / 2}$ state. This reduces the average dead time for the measurement cycle. The ${ }^{2} P_{3 / 2}$ state decays to the ${ }^{2} S_{1 / 2}$ state 350 times more frequently than to the ${ }^{2} D_{5 / 2}$ state, ${ }^{21}$ so there is very little possibility that the ion will return to the ${ }^{2} D_{5 / 2}(F=3)$, which is not emptied by the laser.

The additional laser beams have reduced the average dead time per measurement cycle from around $80 \mathrm{~ms}$ to around $20 \mathrm{~ms}$. The resonance period is typically $40 \mathrm{~ms}$, so the duty cycle has improved from $33 \%$ to $66 \%$. The remaining dead time is due largely to the fluorescence detection time of about $10 \mathrm{~ms}$ and could be reduced further.

\subsection{Laser Frequency Servo}

In order to lock the frequency of the clock laser to the center of the $282 \mathrm{~nm}$ transition, the probability for driving the clock transition is measured for frequencies slightly above and both slightly below the estimated resonance frequency. If the transition probability for excitation on the high-frequency side is denoted by $P(H)$ and for excitation on the low-frequency side by $P(L)$, then a measurement result of $P(H)>P(L)$ indicates that the laser frequency is too low, and vice versa. The signal-to-noise ratio is limited fundamentally by quantum projection noise, due to the fact that the atom is found to be in one state or the other when measured, rather than in some superposition. ${ }^{22}$ This means that several measurements must be averaged in order to reliably determine the difference between the laser and the clock transition. Two possible sources of frequency error of the locked laser are drift of the signal amplitude and drift of the resonant frequency of the Fabry-Pérot cavity to which the laser is locked. The algorithm for the laser frequency servo attempts to address both issues.

\subsubsection{Amplitude drift cancelation}

Drift of the signal amplitude might be caused by drift in the laser intensity at the position of the ion. If the intensity drifts downward in time, and if the servo error signal is derived from a high-frequency measurement followed by a low-frequency measurement $(H L)$ then the servo algorithm would cause the laser frequency to be set too low. If the signal drift is linear with time, then this can be compensated by following the $(H L)$ sequence with a $(L H)$ sequence and averaging the results. That is, a linear signal drift is compensated by deriving the error signal from a $(H L L H)$ or $(L H H L)$ sequence of measurements. It has been shown that this method can be generalized to compensate for drifts having arbitrary polynomial time dependence. ${ }^{23}$ The error signal for the $\mathrm{Hg}^{+}$frequency servo is derived from the $(H L L H L H H L)$ sequence, which compensates for linear or quadratic signal drifts. Since several measurements must be made in order to reduce the amount of projection noise, there is essentially no cost to using this sequence.

\subsubsection{Cavity drift compensation}

The clock laser is stabilized to a vibrationally-isolated Fabry-Pérot cavity. ${ }^{8}$ While this results in sub-hertz laser linewidths, long-term drifts of the locked laser frequency of around $1 \mathrm{~Hz} / \mathrm{s}$ are observed, and the drift rate can change significantly during an experiment. If a simple servo algorithm is used, then the drift will lead to a frequency error, dependent on the servo gain, because the servo will never quite catch up to the atomic resonance frequency. In general, local-oscillator frequency drifts of this sort are compensated by introducing another stage of integration into the servo response function. 
In the $\mathrm{Hg}^{+}$frequency servo, the additional integration stage is implemented by introducing a chirped radiofrequency oscillator whose frequency is added to that of the clock laser with an acousto-optic modulator (AOM). The chirped oscillator consists of a frequency synthesizer whose frequency can be changed, under computer control, while maintaining phase continuity. If the drift rate of the cavity does not match the rate of frequency change of the chirped frequency synthesizer, then the frequency corrections made by the servo algorithm will tend to be all in the same direction, and the frequency of the locked laser (relative to the cavity resonance frequency) will change linearly with time. Periodically, the computer controlling the frequency servo performs a least-squares fit to the record of frequency corrections and modifies the rate of frequency change of the chirped frequency synthesizer in order to decrease the mismatch.

\subsection{Averaging of the Quadrupole Shift}

Previously, the uncertainty of the frequency of the $\mathrm{Hg}^{+}$optical frequency standard was dominated by the uncertainty of the electric quadrupole shift. ${ }^{14}$ This shift comes about because the electronic charge density of the ${ }^{2} D_{5 / 2}$ state has an electric quadrupole moment, which leads to an energy shift if a static electric field gradient is present. Although no static electric field gradient is applied deliberately, small, uncontrolled electric field gradients might be present, and would be difficult to detect.

The ${ }^{2} D_{5 / 2}$ electric quadrupole moment $\Theta$ was measured by observing the shift of the clock frequency when applying an electric field gradient. ${ }^{24}$ We found that $\Theta=(-0.510 \pm 0.018) e a_{0}^{2}$, where $e$ is the elementary charge and $a_{0}$ is the Bohr radius. When no electric field gradient was deliberately applied, the fractional shift of the clock frequency was less than $1 \times 10^{-16}$. A multi-configuration Dirac-Hartree-Fock calculation gave the result $\Theta=-0.564 e a_{0}^{2}$, which disagrees with the experiment by about $10 \% \cdot{ }^{25}$ Recently, $\Theta$ has been calculated by the Fock-state unitary coupled-cluster theory to be $-0.517 e a_{0}^{2}$, in good agreement with the experiment. ${ }^{26}$

It is actually not necessary to know the value of the electric quadrupole moment in order to eliminate its effect on the frequency standard. At least two methods are available for canceling the quadrupole shift, both of which make use of the symmetries of the electric quadrupole interaction, but do not depend on its magnitude. First, the shift is zero when averaged over any three mutually perpendicular quantization axes. ${ }^{27}$ Second, the shift vanishes when an average over $m_{F}$ components is done. ${ }^{28}$

The $\mathrm{Hg}^{+}$frequency standard makes use of the first of the two methods in order to eliminate the quadrupole shift. The orientation of the static magnetic field is switched among three mutually orthogonal directions, so that an equal amount of time is spent at each orientation. ${ }^{16}$ The remaining fractional frequency uncertainty of about $1 \times 10^{-17}$ is due to the uncertainty of the magnetic field orientations.

\subsection{Other Systematic Uncertainties}

The total fractional systematic uncertainty of the $\mathrm{Hg}^{+}$clock frequency is $3.2 \times 10^{-17}$. The various contributions to the systematic uncertainty have been discussed previously. ${ }^{16}$ Some of these contributions have been reduced in recent work. Here we discuss the most important contributions in general terms. The fractional secondorder Doppler shift due to thermal motion is less than $1 \times 10^{-17}$, because the ion is laser cooled to near the Doppler cooling limit. The fractional second-order Doppler shift due to rf micromotion is also less than $1 \times 10^{-17}$, because the stray electric fields that lead to excess micromotion are compensated by use of an rf-phasesensitive fluorescence detection method. ${ }^{29}$ The static magnetic field is periodically measured by interrupting the frequency servo and observing the resonance line of a first-order magnetic-field dependent Zeeman component of the ${ }^{2} S_{1 / 2}(F=0)$ to ${ }^{2} D_{5 / 2}(F=2)$ line. The uncertainty of the fractional shift due to variations in the static magnetic field is less than $1 \times 10^{-17}$. The blackbody radiation shift is negligible because the trap is operated at liquid helium temperature, about $4.2 \mathrm{~K}$. An AC Zeeman shift due to unbalanced rf currents in the trap electrodes has been considered. This fractional shift is estimated to be less than $3 \times 10^{-17}$.

\section{$2.5 \mathrm{Hg}^{+}$Optical to Cs Microwave Frequency Comparison}

Because the SI second is based on the frequency of the cesium ground-state hyperfine transition, making an absolute frequency measurement at the highest level of accuracy requires a primary cesium frequency standard as a reference. The frequency of a laser frequency-locked to the $\mathrm{Hg}^{+}$clock frequency can be compared to a microwave frequency by using a self-referenced femtosecond laser frequency comb. ${ }^{10-12}$ The NIST-F1 cesium 


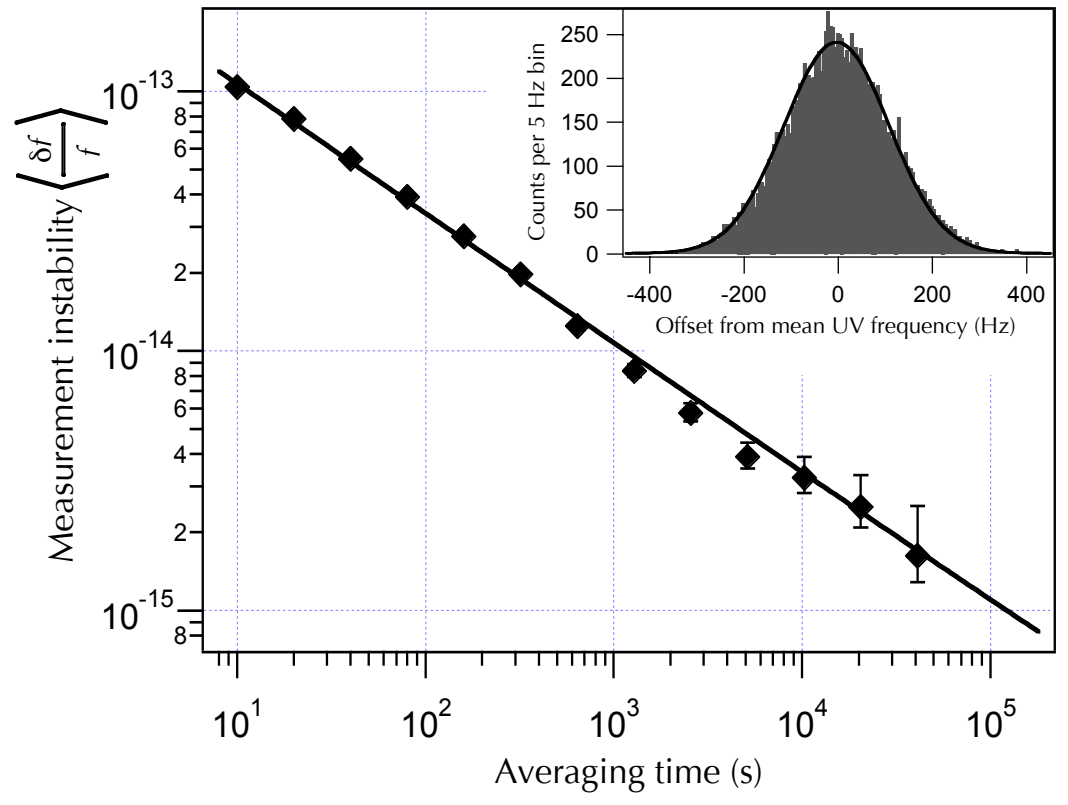

Figure 2. Instability of the ratio of the $\mathrm{Hg}^{+}$clock frequency relative to the cesium frequency standard. The quantity plotted is the Total Deviation. The inset is a histogram of the frequency values and a fitted Gaussian function.

atomic fountain has a fractional frequency uncertainty of around $4 \times 10^{-16} \cdot{ }^{30}$ Comparisons have been made over several years between NIST-F1 and the $\mathrm{Hg}^{+}$optical frequency standard. ${ }^{14-18}$ The results of a series of measurements of the $\mathrm{Hg}^{+}$frequency, referenced to NIST-F1, are shown in Fig. 2. ${ }^{16}$ The Total Deviation is plotted as a function of the averaging time. The Total Deviation is similar to the better-known Allan deviation, but is a better predictor of long-term fractional frequency instability. ${ }^{31}$ The inset is a histogram of the optical frequency measurements, together with a fitted Gaussian function. A recent evaluation of all measurements gives the value of the $\mathrm{Hg}^{+}$frequency $f\left(\mathrm{Hg}^{+}\right)=1064721609899145.30 \pm 0.69 \mathrm{~Hz}$. The fractional frequency uncertainty of $6.5 \times 10^{-16}$ is within a factor of 1.5 of the uncertainty of the NIST-F1 frequency standard. ${ }^{18}$

\section{ALUMINUM ION FREQUENCY STANDARD}

The possibility of using the transitions from the ground ${ }^{1} S_{0}$ state to the metastable ${ }^{3} P_{0}$ state in ${ }^{27} \mathrm{Al}^{+}$and other group IIIA ions for an optical frequency standard was first pointed out by Dehmelt. ${ }^{32,33}$ These transitions have extremely high $Q \mathrm{~s}$ and have the additional advantage of having no electric quadrupole shift, since a $J=0$ state has zero electric quadrupole moment.

The energy levels of ${ }^{27} \mathrm{Al}^{+}$that are relevant to the operation of the frequency standard are shown in Fig. 3. While the strongly allowed $167 \mathrm{~nm} 3 s^{2}{ }^{1} S_{0}$ to $3 s 3 p{ }^{1} P_{1}$ transition would be useful for laser cooling and state detection, narrowband tunable lasers are not available at that wavelength.

To get around this problem, Wineland proposed to simultaneously trap an auxiliary ion, which could be laser cooled and optically detected at a more convenient wavelength. ${ }^{34}$ Because the two ions are coupled through the Coulomb interaction, the "clock" ion (e. g., ${ }^{27} \mathrm{Al}^{+}$) is also cooled. Further, the superposition state of the clock ion can be transferred to the auxiliary ion, making use of the fact that they share a vibrational degree of freedom. That is, if the state of the clock ion is $(\alpha|S\rangle+\beta|P\rangle)$, the state of the auxiliary ion becomes $(\alpha|1\rangle+\beta|2\rangle)$, where $|1\rangle$ and $|2\rangle$ are two of the hyperfine ground state sublevels. This makes it possible to detect whether the clock ion has been driven to the metastable state by observing the fluorescence of the auxiliary ion.

The basic methods were demonstrated at NIST with a ${ }^{27} \mathrm{Al}^{+}$clock ion and a ${ }^{9} \mathrm{Be}^{+}$auxiliary ion. ${ }^{35} \mathrm{The}^{27} \mathrm{Al}^{+}$ ${ }^{1} S_{0}$ to ${ }^{3} P_{1}$ transition (A in Fig. 3) was used for this demonstration. This transition has a natural linewidth of about $500 \mathrm{~Hz},{ }^{36}$ so it is not the best choice for an optical frequency standard. More recently, the ${ }^{27} \mathrm{Al}^{+}{ }^{1} S_{0}$ to 


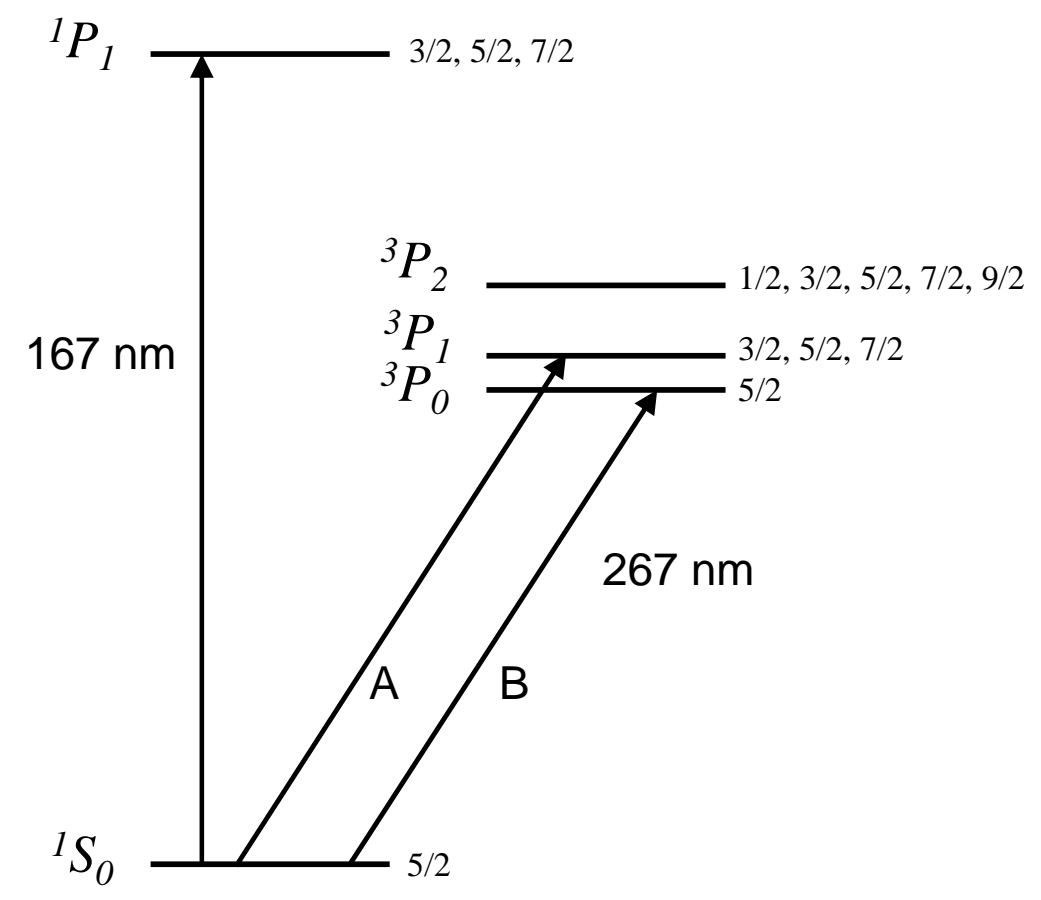

Figure 3. Energy levels of ${ }^{27} \mathrm{Al}^{+}$. Numbers to the right of the energy levels are the values of $F$, the total angular momentum quantum number. The energy separations between hyperfine energy levels are not shown. The strong transition at 167 $\mathrm{nm}$ is not directly driven, for lack of a tunable laser at that wavelength. Transitions near $267 \mathrm{~nm}$ induced by the lasers are labeled $\mathrm{A}$ and $\mathrm{B}$. The energy differences between the ${ }^{3} P_{J}$ fine-structure levels are expanded for clarity.

${ }^{3} P_{0}$ transition (B in Fig. 3) has been observed. ${ }^{37,38}$ This transition is the basis for the ${ }^{27} \mathrm{Al}^{+}$optical frequency standard discussed here. Detection of the ${ }^{1} S_{0}$ to ${ }^{3} P_{0}$ transition depends on the previously demonstrated ${ }^{27} \mathrm{Al}^{+}$ to ${ }^{9} \mathrm{Be}^{+}$quantum state transfer. Details of the methods used to frequency-lock a laser to the ${ }^{9} \mathrm{Be}^{+1} S_{0}$ to ${ }^{3} P_{0}$ resonance have been published. ${ }^{37}$

\subsection{Atomic System}

In a nonrelativistic approximation, the $3 s 3 p{ }^{3} P_{1}$ state does not decay to the ground state, while the $3 s 3 p{ }^{1} P_{1}$ decay is fully allowed. However, spin-orbit and other relativistic interactions mix the $3 s 3 p{ }^{3} P_{1}$ and $3 s 3 p{ }^{1} P_{1}$ states and allow both states to decay. In the absence of hyperfine interaction, the $3 s 3 p^{3} P_{0}$ state does not decay by any single-photon process. However, the hyperfine interaction mixes the $3 s 3 p{ }^{3} P_{0}$ state with other states, mainly the $3 s 3 p{ }^{1} P_{1}$ and $3 s 3 p{ }^{3} P_{1}$ states, that do decay to the ground state. This makes the $3 s 3 p{ }^{3} P_{0}$ to $3 s^{2}{ }^{1} S_{0}$ decay weakly allowed. ${ }^{39}$ The lifetime of the ${ }^{3} P_{0}$ state was measured to be $20.6 \pm 1.4 \mathrm{~s}$, so the transition has a natural $Q$ of $1.45 \times 10^{17}$.

Since the nuclear spin of ${ }^{27} \mathrm{Al}$, the only stable isotope of aluminum, has spin $I=5 / 2$, both the ${ }^{1} S_{0}$ and the ${ }^{3} P_{0}$ state have total angular momentum $F=5 / 2$. Therefore, each $m_{F}$ component of the ${ }^{1} S_{0}$ and ${ }^{3} P_{0}$ states has a linear Zeeman shift. In the absence of hyperfine-induced mixing between $J$ states, the $g$ factors of the ground and excited states would be nearly equal to each other and to the nuclear $g$ factor. This would make the frequencies of the ${ }^{1} S_{0}\left(F=5 / 2, m_{F}\right)$ to ${ }^{3} P_{0}\left(F=5 / 2, m_{F}\right)$ transitions nearly independent of magnetic field. In fact there is a large shift of the $g$ factor of the ${ }^{3} P_{0}$ state compared to that of the ${ }^{1} S_{0}$ state that has recently been measured. ${ }^{37}$ It was found that $g\left({ }^{3} P_{0}\right)=-0.00197686(21)$ and $g\left({ }^{1} S_{0}\right)=-0.00079248(14)$, where the $g$ factors are defined in terms of the Bohr magneton, and the numbers in parentheses are the uncertainties in units of the least significant digits. The shift of $g\left({ }^{3} P_{0}\right)$ relative to $g\left({ }^{1} S_{0}\right)$ is due to the hyperfine interaction mixing the ${ }^{3} P_{0}$ with other other $J$ states, mainly $3 s 3 p^{3} P_{1}$. This type of $g$ factor shift was first observed in the $6 s 6 p^{3} P_{0}$ states of ${ }^{199} \mathrm{Hg}$ and ${ }^{201} \mathrm{Hg} .{ }^{40}$ 


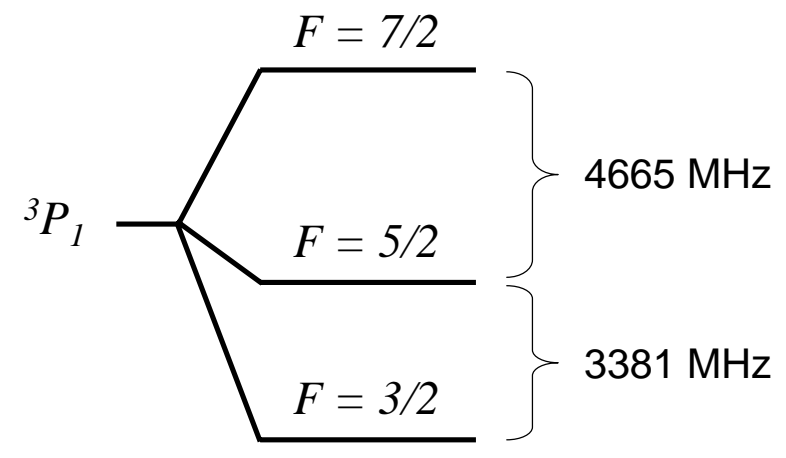

Figure 4. Hyperfine structure of the $6 s 6 p^{3} P_{1}$ state of ${ }^{27} \mathrm{Al}^{+}$.

\subsection{Comparison of Atomic Calculations with Experiment}

Recently, some calculations of diagonal and off-diagonal hyperfine constants of ${ }^{27} \mathrm{Al}^{+}$have been carried out. ${ }^{41}$ The GRASP92 set of programs ${ }^{42}$ was used to generate the atomic state functions for the $\left\{{ }^{1} P_{1},{ }^{3} P_{2},{ }^{3} P_{1},{ }^{3} P_{0}\right\}$ set of states by the multi-configuration Dirac-Hartree-Fock method, and the HFS92 program ${ }^{43}$ was used to calculate the magnetic dipole $(A)$ and electric quadrupole $(B)$ hyperfine constants. Values for the nuclear magnetic moment $\mu_{I}=3.64150687(65) \mu_{N}$ and electric quadrupole moment $Q=0.1466(10) \times 10^{-24} \mathrm{e} \mathrm{cm}^{2}$ were assumed. ${ }^{44,45}$ The results are listed in Table 1.

These calculations can be connected with experiment in at least three different ways:

First, the hyperfine separations of the ${ }^{3} P_{1}$ state have been measured at NIST (see Fig. 4). The separations were measured to be $4664.903(1) \mathrm{MHz}$ and $3380.688(1) \mathrm{MHz} .{ }^{46}$ The uncertainties are due mainly to the uncertainty of the quadratic Zeeman shift, since the measurements were made at nonzero magnetic field. Extraction of the diagonal $A$ and $B$ coefficients is not straightforward, because the second-order magnetic dipole energy is comparable to the first-order electric quadrupole energy. One way to test the calculation is to compare the predicted separations, calculated to second order in perturbation theory, with the observed separations. The predicted separations are $4693 \mathrm{MHz}$ and $3400 \mathrm{MHz}$, which disagree with experiment by less than $2 \%$.

Second, we can use the off-diagonal $A$ coefficients that mix the ${ }^{3} P_{0}$ state with the ${ }^{1} P_{1}$ and ${ }^{3} P_{1}$ states to calculate the radiative decay rate of the ${ }^{3} P_{0}$ state. ${ }^{47}$ This calculation also requires the radiative lifetimes of the ${ }^{3} P_{1}$ state and the ${ }^{1} P_{1}$ state. We take the former from experiment ${ }^{36}$ and the latter from the NIST database. ${ }^{48}$ The result is $22.7 \mathrm{~s}$, compared to the experimental result of $20.6 \pm 1.6 \mathrm{s.}^{37}$

Third, we can use the same off-diagonal $A$ coefficients to calculate the difference between the $g$ factors of the ${ }^{3} P_{0}$ and ${ }^{1} S_{0}$ states. ${ }^{47}$ The result is $g\left({ }^{3} P_{0}\right)-g\left({ }^{1} S_{0}\right)=-1.181 \times 10^{-3}$, compared to the experimental result -1.184 $37(8) \times 10^{-3} .37$

Thus, it appears that calculational method is capable of reproducing the experimental hyperfine constants to within a few percent. The prediction of the ${ }^{3} P_{0}$ decay rate has a larger error, but may be due to the uncertainties of the ${ }^{1} P_{1}$ and ${ }^{3} P_{1}$ lifetimes.

\subsection{Systematic Uncertainties}

Our current estimate of the fractional systematic uncertainty of the $\mathrm{Al}^{+}$clock frequency is less than $5 \times 10^{-17}$. $^{49}$ The greatest part of the systematic uncertainty is due to the second-order Doppler shift, from both the thermal motion and the micromotion. The linear Zeeman shift is cancelled by alternately observing the ${ }^{1} S_{0}(F=$ $\left.5 / 2, m_{F}=+5 / 2\right)$ to ${ }^{3} P_{0}\left(F=5 / 2, m_{F}=+5 / 2\right)$ and the ${ }^{1} S_{0}\left(F=5 / 2, m_{F}=-5 / 2\right)$ to ${ }^{3} P_{0}\left(F=5 / 2, m_{F}=-5 / 2\right)$ transitions and averaging the frequencies. The quadratic Zeeman shift has been measured, and it contributes less than $1 \times 10^{-18}$ to the fractional frequency uncertainty.

The blackbody radiation shift is unusually small for an optical frequency standard because of a fortuitous cancelation between the ${ }^{1} S_{0}$ and ${ }^{3} P_{0}$ quadratic Stark shifts. ${ }^{50}$ The fractional frequency shift at $300 \mathrm{~K}$ is $8(5) \times$ $10^{-18}$. At the normal operating temperature of the frequency standard, it is $12(5) \times 10^{-18}$. 
Table 1. Calculated values of the diagonal and off-diagonal hyperfine $A$ and $B$ constants within the $3 s 3 p{ }^{1,3} P_{J}$ set of states.

\begin{tabular}{|c|c|r|r|}
\hline$\Gamma$ & $\Gamma^{\prime}$ & $\begin{array}{r}A\left(\Gamma, \Gamma^{\prime}\right) \\
(\mathrm{MHz})\end{array}$ & $\begin{array}{r}B\left(\Gamma, \Gamma^{\prime}\right) \\
(\mathrm{MHz})\end{array}$ \\
\hline \hline${ }^{3} P_{2}$ & ${ }^{3} P_{2}$ & 1149 & 31.42 \\
${ }^{3} P_{2}$ & ${ }^{3} P_{1}$ & -539 & 7.84 \\
${ }^{3} P_{2}$ & ${ }^{1} P_{1}$ & 845 & -0.05 \\
${ }^{3} P_{2}$ & ${ }^{3} P_{0}$ & 0 & 13.57 \\
${ }^{3} P_{1}$ & ${ }^{3} P_{1}$ & 1348 & -15.62 \\
${ }^{3} P_{1}$ & ${ }^{1} P_{1}$ & 1571 & 0.18 \\
${ }^{3} P_{1}$ & ${ }^{3} P_{0}$ & -1320 & 0 \\
${ }^{1} P_{1}$ & ${ }^{3} P_{0}$ & -1045 & 0 \\
\hline
\end{tabular}

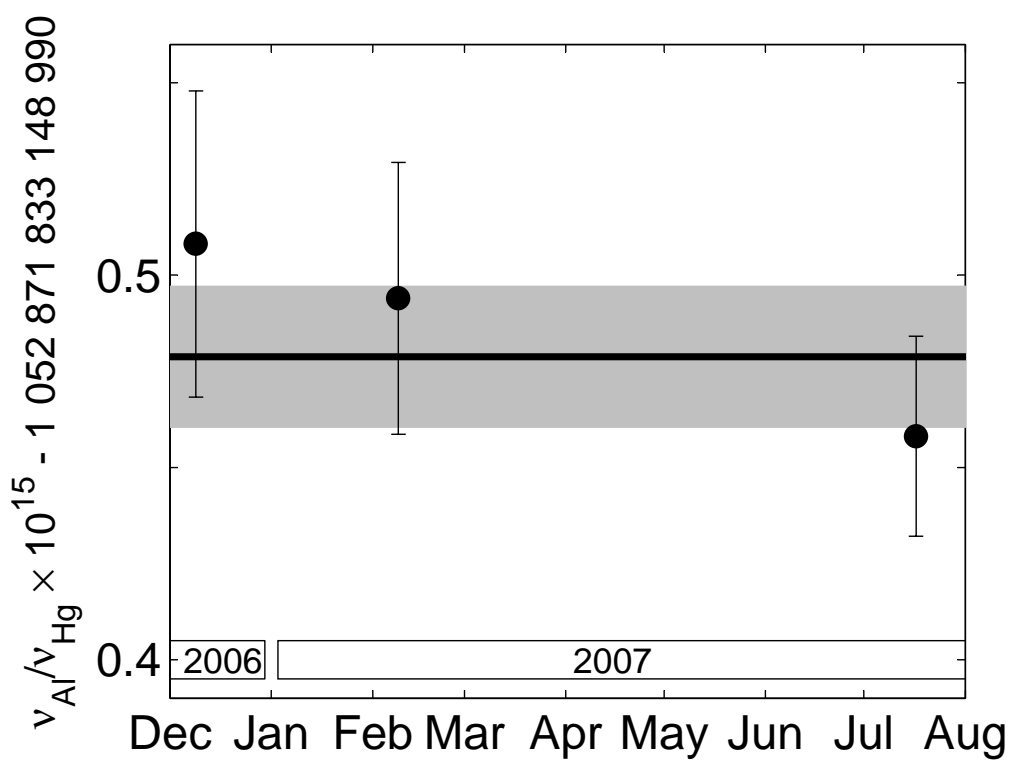

Figure 5. Measurements of the ratio of the $\mathrm{Al}^{+}$and $\mathrm{Hg}^{+}$clock frequencies as a function of the date the measurements were made. 


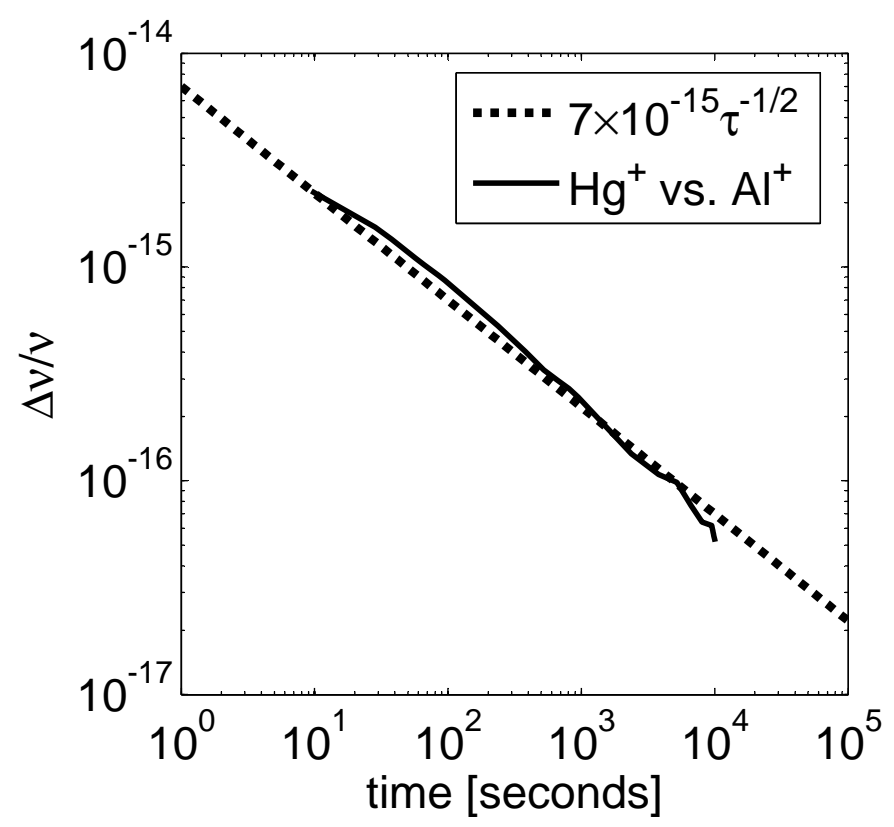

Figure 6. Instability of the ratio of the $\mathrm{Al}^{+}$and $\mathrm{Hg}^{+}$clock frequencies. The quantity plotted is the Allan deviation, the square root of the Allan variance.

While to first order, the ${ }^{3} P_{0}$ state has zero electric quadrupole moment, the hyperfine interaction mixes this state with states having $J=1,2$, such as the nearest ${ }^{3} P_{1}$ and ${ }^{3} P_{2}$ states. This results in an electronic quadrupole moment of about $-1.2 \times 10^{-5} \mathrm{ea}_{0}^{2}{ }^{41}$ This quadrupole can interact with the electric field gradients due to the ion trap and to the presence of the ${ }^{9} \mathrm{Be}^{+}$ion, resulting in a fractional frequency shift that is less than $1 \times 10^{-18}$ and can be neglected.

\section{4. $\mathrm{HG}^{+}-\mathrm{AL}^{+}$FREQUENCY COMPARISONS}

The $\mathrm{Hg}^{+}$and $\mathrm{Al}^{+}$frequency standards have been operated simultaneously. The frequency of one mode or "tooth" of the self-referenced femtosecond laser frequency comb can be phase-locked to the frequency of one standard. The frequency of the heterodyne beat-note of the other frequency standard with the nearest comb tooth is measured. The measurement can be converted to the frequency ratio of the two standards and does not depend on the accuracy of any microwave frequency standards used as references. ${ }^{51}$ Figure 5 is a plot of measurements of the ratio of the frequency of the $\mathrm{Al}^{+}$standard to that of the $\mathrm{Hg}^{+}$standard. The reproducibility of the ratio is seen to be better than $1 \times 10^{-16}$, which is better than the accuracy of the primary cesium frequency standard. Figure 6 shows the fractional frequency instability of the ratio as a function of averaging time.

\section{ACKNOWLEDGMENTS}

This work was partially supported by both the Office of Naval Research and NIST, agencies of the U. S. government; it is not subject to U. S. copyright.

\section{REFERENCES}

1. P. L. Bender, J. L. Hall, R. H. Garstang, F. M. J. Pichanick, W. W. Smith, R. L. Barger, and J. B. West Bull. Am. Phys. Soc. 21, p. 599, 1976.

2. J. C. Bergquist, D. J. Wineland, W. M. Itano, H. Hemmati, H.-U. Daniel, and G. Leuchs Phys. Rev. Lett. 55, p. $1567,1985$. 
3. J. C. Bergquist, R. G. Hulet, W. M. Itano, and D. J. Wineland Phys. Rev. Lett. 57, p. 1699, 1986.

4. W. M. Itano, J. C. Bergquist, R. G. Hulet, and D. J. Wineland Phys. Rev. Lett. 59, p. 2732, 1987.

5. A. G. Calamai and C. E. Johnson Phys. Rev. A 42, p. 5425, 1990.

6. J. C. Bergquist, W. M. Itano, and D. J. Wineland Phys. Rev. A 36, p. 428, 1987.

7. R. H. Dicke Phys. Rev. 89, p. 472, 1953.

8. B. C. Young, F. C. Cruz, W. M. Itano, and J. C. Bergquist Phys. Rev. Lett. 82, p. 3799, 1999.

9. R. J. Rafac, B. C. Young, J. A. Beall, W. M. Itano, D. J. Wineland, and J. C. Bergquist Phys. Rev. Lett. 85, p. $2462,2000$.

10. S. A. Diddams, D. J. Jones, J. Ye, S. T. Cundiff, J. L. Hall, J. K. Ranka, R. S. Windeler, R. Holzwarth, T. Udem, and T. W. Hänsch Phys. Rev. Lett. 84, p. 5102, 2000.

11. D. J. Jones, S. A. Diddams, J. K. Ranka, A. Stentz, R. S. Windeler, J. L. Hall, and S. T. Cundiff Science 288, p. $635,2000$.

12. R. Holzwarth, T. Udem, T. W. Hänsch, J. C. Knight, W. J. Wadsworth, and P. S. J. Russell Phys. Rev. Lett. 85, p. 2264, 2000.

13. S. A. Diddams, T. Udem, J. C. Bergquist, E. A. Curtis, R. E. Drullinger, L. Hollberg, W. M. Itano, W. D. Lee, C. W. Oates, K. R. Vogel, and D. J. Wineland Science 293, p. 825, 2001.

14. T. Udem, S. A. Diddams, K. R. Vogel, C. W. Oates, E. A. Curtis, W. D. Lee, W. M. Itano, R. E. Drullinger, J. C. Bergquist, and L. Hollberg Phys. Rev. Lett. 86, p. 4996, 2001.

15. S. Bize, S. A. Diddams, U. Tanaka, C. E. Tanner, W. H. Oskay, R. E. Drullinger, T. E. Parker, T. P. Heavner, S. R. Jefferts, L. Hollberg, W. M. Itano, and J. C. Bergquist Phys. Rev. Lett. 90, p. 150802, 2003.

16. W. H. Oskay, S. A. Diddams, E. A. Donley, T. M. Fortier, T. P. Heavner, L. Hollberg, W. M. Itano, S. R. Jefferts, M. J. Delaney, K. Kim, F. Levi, T. E. Parker, and J. C. Bergquist Phys. Rev. Lett. 97, p. 020801, 2006.

17. T. M. Fortier, N. Ashby, J. C. Bergquist, M. J. Delaney, S. A. Diddams, T. P. Heavner, L. Hollberg, W. M. Itano, S. R. Jefferts, K. Kim, F. Levi, L. Lorini, W. H. Oskay, T. E. Parker, J. Shirley, and J. E. Stalnaker Phys. Rev. Lett. 98, p. 070801, 2007.

18. J. E. Stalnaker, S. A. Diddams, T. M. Fortier, L. Hollberg, J. C. Bergquist, W. M. Itano, M. J. Delaney, L. Lorini, W. H. Oskay, T. P. Heavner, S. R. Jefferts, F. Levi, T. E. Parker, and J. Shirley Appl. Phys. B, (in press).

19. D. J. Berkeland and M. G. Boshier Phys. Rev. A 65, p. 033413, 2002.

20. D. J. Berkeland, J. D. Miller, J. C. Bergquist, W. M. Itano, and D. J. Wineland Phys. Rev. Lett. 80, p. 2089, 1998.

21. D. H. Crandall, R. A. Phaneuf, and G. H. Dunn Phys. Rev. A 11, p. 1223, 1975.

22. W. M. Itano, J. C. Bergquist, J. J. Bollinger, J. M. Gilligan, D. J. Heinzen, F. L. Moore, M. G. Raizen, and D. J. Wineland Phys. Rev. A 47, p. 3554, 1993.

23. G. E. Harrison, M. A. Player, and P. G. H. Sandars J. Phys. E 4, p. 750, 1971.

24. W. H. Oskay, W. M. Itano, and J. C. Bergquist Phys. Rev. Lett. 94, p. 163001, 2005.

25. W. M. Itano Phys. Rev. A 73, p. 022510, 2006.

26. C. Sur and R. K. Chaudhuri Phys. Rev. A , (in press); preprint: arXiv:0707.3587v2.

27. W. M. Itano J. Research National Institute of Standards and Technology 105, p. 829, 2000.

28. P. Dubé, A. A. Madej, J. E. Bernard, L. Marmet, J.-S. Boulanger, and S. Cundy Phys. Rev. Lett. 95, p. 033001, 2005.

29. D. J. Berkeland, J. D. Miller, J. C. Bergquist, W. M. Itano, and D. J. Wineland J. Appl. Phys. 83, p. 5025, 1998.

30. T. P. Heavner, S. R. Jefferts, E. A. Donley, J. H. Shirley, and T. E. Parker Metrologia 42, p. 411, 2005.

31. C. A. Greenhall, D. A. Howe, and D. B. Percival IEEE Trans. Ultrason. Ferroelectr. Freq. Control 46, p. 1183, 1999.

32. H. Dehmelt Bull. Am. Phys. Soc. 20, p. 60, 1975.

33. H. G. Dehmelt IEEE Trans. Instrum. Meas. 31, p. 83, 1982. 
34. D. J. Wineland, J. C. Bergquist, J. J. Bollinger, R. E. Drullinger, and W. M. Itano in Proceedings of the 6th Symposium on Frequency Standards and Metrology, P. Gill, ed., pp. 361-368, World Scientific, Singapore, 2002.

35. P. O. Schmidt, T. Rosenband, C. Langer, W. M. Itano, J. C. Bergquist, and D. J. Wineland Science 309, p. 749, 2005.

36. E. Träbert, A. Wolf, J. Linkemann, and X. Tordoir J. Phys. B 32, p. 537, 1999.

37. T. Rosenband, P. O. Schmidt, D. B. Hume, W. M. Itano, T. M. Fortier, J. E. Stalnaker, K. Kim, S. A. Diddams, J. C. J. Koelemeij, J. C. Bergquist, and D. J. Wineland Phys. Rev. Lett. 98, p. 220801, 2007.

38. D. B. Hume, T. Rosenband, and D. J. Wineland Phys. Rev. Lett. , (in press); preprint: arXiv:0705.1870v2.

39. T. Brage, P. G. Judge, A. Aboussaïd, M. R. Godefroid, P. Jönsson, A. Ynnerman, C. F. Fischer, and D. S. Leckrone Astrophys. J. 500, p. 507, 1998.

40. B. Lahaye and J. Margerie J. Phys. (Paris) 36, p. 943, 1975.

41. W. M. Itano (unpublished calculation).

42. F. A. Parpia, C. F. Fischer, and I. P. Grant Comput. Phys. Commun. 94, p. 249, 1996.

43. P. Jönsson, F. A. Parpia, and C. F. Fischer Comput. Phys. Commun. 96, p. 301, 1996.

44. P. Raghavan At. Data Nucl. Data Tables 42, p. 189, 1989.

45. P. Pyykkö Mol. Phys. 99, p. 1617, 2001.

46. T. Rosenband et al. (unpublished work).

47. E. Peik, G. Hollemann, and H. Walther Phys. Rev. A 49, p. 402, 1994.

48. "NIST Atomic Spectra Database," http://physics.nist.gov/PhysRefData/ASD/index.html.

49. T. Rosenband et al. (manuscript in preparation).

50. T. Rosenband, W. M. Itano, P. O. Schmidt, D. B. Hume, J. C. J. Koelemeij, J. C. Bergquist, and D. J. Wineland in Proc. 20th European Time and Frequency Forum, pp. 289-292, 2006; preprint: arXiv:physics/0611125v2.

51. L.-S. Ma, Z. Bi, A. Bartels, L. Robertsson, M. Zucco, R. S. Windeler, G. Wilpers, C. Oates, L. Hollberg, and S. A. Diddams Science 303, p. 1843, 2004. 